\title{
Roles of ecdysteroids for progression of reproductive cycle in the fresh water crustacean Daphnia magna
}

\author{
Eri Sumiya ${ }^{1,2}$, Yukiko Ogino ${ }^{1,2 \dagger}$, Hitoshi Miyakawa ${ }^{2 \dagger}$, Chizue Hiruta ${ }^{2}$, Kenji Toyota ${ }^{1,2}$, Shinichi Miyagawa ${ }^{1,2}$ \\ and Taisen Iguchi ${ }^{1,2^{*}}$
}

\begin{abstract}
Introduction: Daphnia magna exhibits a parthenogenetic reproductive cycle linked to a moulting cycle, but regulatory mechanisms of neither moulting nor reproductive cycle are understood in daphnids. Moulting is regulated by ecdysteroids in insects. A previous study showed that a titre of ecdysteroids changed during the reproductive cycle in D. magna; however, no clear correlation among titre, moulting and reproductive cycles has been proved in daphnids. To understand endocrine mechanisms underlying the coordinated reproductive cycle, we analysed the expression of genes coding for enzymes in ecdysteroids synthesis or inactivation pathways, and the effects of 20-hydroxyecdysone (20E) on moulting and ovulation in D. magna.

Results: We cloned orthologues of neverland ( $n v d$ ) and shade (shd) in the ecdysteroids synthesis pathway, and Cyp18a1 in the ecdysteroids inactivation pathway previously identified in insects. Gene expression of Cyp18a1 changed conversely with the fluctuation in ecdysteroids titre during the intermoulting period. Tissue-specific expression analysis of nvd showed a prominent expression in the gut. Furthermore, treatment of adult female D. magna with 20E inhibited moulting and/or ovulation.
\end{abstract}

Conclusions: Our cloning and phylogenetic analyses showed that nvd and shd as well as Cyp 18a1 are evolutionary conserved in D. magna, suggesting that these genes appeared in arthropods before the radiation of insects. The gene expression analysis during the reproductive cycle indicated that Cyp18a1 possibly regulates the decline of ecdysteroid titre before moulting and ovulation. Furthermore, the expression of nvd in the gut suggested that ecdysone might be synthesised in the gut. Exogenous 20E-treatment resulted in the failure of not only moulting, but also ovulation, suggesting that a low level of ecdysteroids before moulting is required for moulting and ovulation in D. magna.

Keywords: Daphnia magna, Ecdysteroids, Moulting, Reproductive cycle, 20-hydroxyecdysone

\section{Introduction}

Crustacea and Insecta comprising monophyletic group, the Pancrustacea, are the most diverse animal group in aquatic habitats [1]. Reproductive diversity as an adaptation to environmental conditions may have facilitated the radiation of crustaceans [2]. Reproduction of decapod crustaceans is regulated by complex endocrine mechanisms

\footnotetext{
* Correspondence: taisen@nibb.ac.jp

${ }^{\dagger}$ Equal contributors

${ }^{1}$ Faculty of Life Science, Graduate University for Advanced Studies (SOKENDAI), 5-1 Higashiyama Myodaiji, Okazaki, Aichi 444-8787, Japan ${ }^{2}$ Okazaki Institute for Integrative Bioscience, National Institute for Basic Biology, National Institutes of Natural Sciences, and Department of Basic Biology, 5-1 Higashiyama Myodaiji, Okazaki, Aichi 444-8787, Japan
}

involving ecdysteroids, neuropeptides, biogenic amines, opioids, juvenile hormones, and vertebrate-type steroids [3]. Daphnids show characteristic features in reproduction. They reproduce parthenogenetically every three days under favourable conditions, and the reproductive cycle occurs synchronously with the moulting cycle. After moulting, mother daphnids ovulate new eggs from the ovary into the brood pouch (ovulation). At the end of each reproductive cycle, the mother releases the brood from the pouch to the surrounding water. After this release, the mother moults again and repeats the cycle. These events occur in a very systematic order with intervals that span approximately three days depending on the condition.

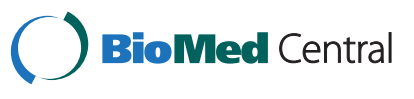


Consequently, a closely related system might regulate the moulting and reproductive cycle. A similar association of moulting with a reproductive cycle has also been reported in other crustaceans such as amphipods, isopods, shrimps, prawns and a sand crab $[4,5]$. However, regulation of the reproductive cycle remains largely unknown in most crustaceans.

Ecdysteroids (ecdysone and 20-hydroxyecdysone; 20E) are steroid hormones that regulate moulting and metamorphosis in insects. These have been extensively studied in insects, and are commonly called "moulting hormones" as their major role implies. Recent studies in insects revealed that ecdysteroids have a variety of functions besides moulting in the control of reproduction and embryogenesis [6]. In Drosophila melanogaster, ecdysteroids are required for differentiation of germ line stem cells $[7,8]$ and prepupal development $[9,10]$. A pioneering study reported that a titre of ecdysteroids increased at the premoult stage and decreased back to the basal level before moulting in Daphnia magna [11], indicating that ecdysteroids are predominant moulting hormones. However, in crustaceans, reports on ecdysteroidal regulation in both moulting and reproduction are scarce [4]. The synchronised moulting and reproductive cycle in daphnids implies a possibility that ecdysteroids may regulate not only moulting, but also the reproductive cycle.

In insects, biosynthesis of ecdysteroids starts with dietary cholesterol. Rieske oxygenase DAF-36 (neverland, nvd) converts it to 7-dehydrocholesterol [12]. After several steps mediated by enzymes belonging to the cytochrome P450 family (CYP450), it is converted to ecdysone. Then, Cyp314A1 (shade, shd) finally converts ecdysone into 20E, which is the biologically active form binding to an ecdysone receptor (EcR) [13,14]. The complex of $20 \mathrm{E}$ and EcR recruits ultraspiracle (USP) in insects and daphnids or retinoid X receptor (RXR) in decapods, and acts as a heterodimer [15]. EcR and USP have been cloned in D. magna [16]. Three EcR paralogues with five distinct N-terminus sequences and one USP were identified [16]. These genes in the ecdysteroids biosynthesis pathway except for $n v d$ belong to CYP450 encoding hydroxylases. D. pulex possesses five CYP450 genes: spook, phantom, disembodied, shadow and shd [17]. In decapod crustaceans, Cyp306A1 (phantom) in kuruma prawn (Marsupenaeus japonicus) [18], and four CYP450s (spook, phantom, disembodied and shadow) and $n v d$ in cray fish (Pontastacus leptodactylus) [19] have been identified to date. However, neither gene expression nor functional analyses have been explored in microcrustaceans such as copepoda, phyllopoda and cladocera [15].

The synthesis and secretion of ecdysone take place in a specialised organ in insects and decapods. Insect larvae synthesise ecdysone in the prothoracic gland although it is destined to degenerate in the adult of most species
[20]. The synthetic organ in decapods is the Y-organ. However, the organ that synthesises and secretes ecdysone has not been identified in microcrustaceans, including daphnids. Some reports have speculated that these species may synthesise ecdysone in the epidermis [15,21,22] since ecdysone production in the epidermis was observed in vitro in the tick Ornithodoros parkeri [23]. Identification of the endocrine organ is essential to understand the endocrine system of daphnids.

As an entry point to understand the roles of ecdysteroids in the reproductive cycle in D. magna, we cloned genes of three key enzymes for 20E synthesis or inactivation: $n v d$, shd and Cyp18a1. Spacio-temporal expression analyses of these genes during the reproductive cycle indicated that ecdysone is possibly synthesised in the gut, and that the decline of the 20E titre before moulting [11] might be regulated by Cyp18a1. In addition, exogenous 20E treatment during the reproductive cycle resulted in a failure of moulting and ovulation, suggesting that a low level of ecdysteroids before moulting is required for successful moulting and ovulation in D. magna. The present study provides insight into the endocrine system that regulates the highly coordinated reproductive system in crustaceans.

\section{Results}

Molecular cloning and phylogenetic analysis of genes in 20E synthesis or inactivation pathway in D. magna

We cloned full-length of $n v d$ encoding 444 amino acids (a.a.), shd encoding 526 a.a. and Cyp18a1 encoding 551 a.a. from $D$. magna. Furthermore, we identified the partial cDNA sequence of a distinct $n v d$. Aligning amino acids of these genes with $D$. pulex and two insect species, D. melanogaster and Bombyx mori, revealed that both $D$. magna nvd contain a characteristic domain, Rieske [2Fe-2S], and a non-heme Fe (II) motif (Figure 1A) [12]. The obtained shd and Cyp18a1 belonging to the P450 family have a P/G-rich domain and five conserved P450 motifs: Helix-C, Helix-I, Helix-K, PERF and Heme-binding (Figure 1B) [24].

Phylogenetic analysis was conducted by comparing the amino acid sequences of the obtained nvd, shd and Cyp18a1 with other available arthropod sequences. In each obtained tree, D. manga and D. pulex clustered together with other arthropods, forming a monophyletic group. This suggests that the orthologous genes of nvd, shd and Cyp18a1 were identified in D. magna. Two of the D. magna nvd were assembled in the Daphnia nvd cluster. These nvd paralogoues were therefore designated nvd1 (full length, AB839171) and nvd2 (partial cDNA sequence, AB839172) (Figure 2A). We further found a duplication of nvd1, nvd1a (sequence ID in $D$. pulex genome database, NCBI_GNO_67514) and nvd1b (NCBI_GNO_462084). 


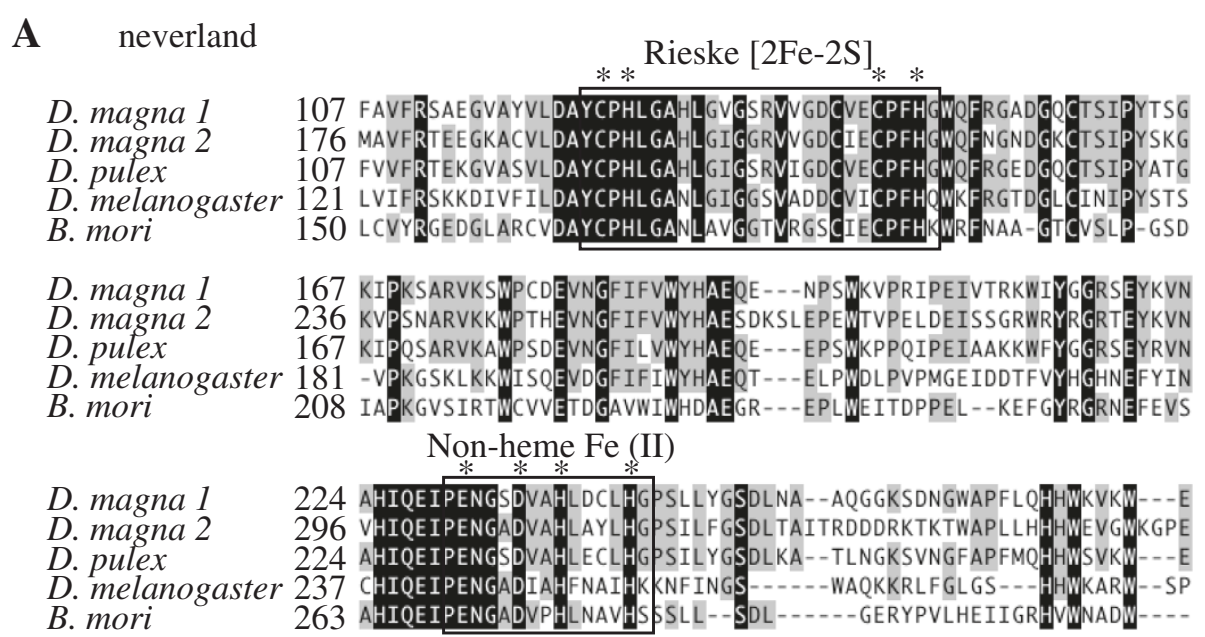

B shade

D. magna

D. pulex

D. melanogaster 58 GIWDIPGPKRIPFLG TKWIFLLFFRRYKMTKLHEVYADLNRQYGDIVLEVMPSNVPIVHL

B. mori

55 HSCRLPGPQPLPFLG RWW---LFWSRYKMNKLHEAYADMFKRYGPVFMETTPGGVAVVSI

Helix-C

D. magna

D. pulex

D. melanogaster 118 YNRDDLEKV

B. mori

112 AERTALEAV
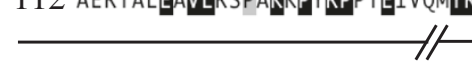

Helix-I

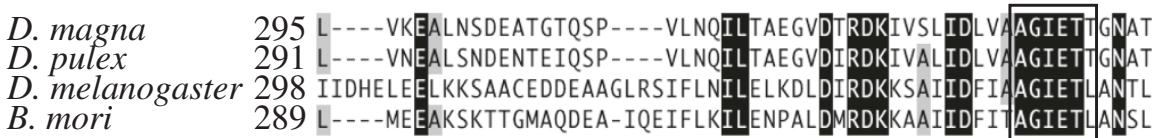

Helix-K

$\begin{array}{ll}\text { D. magna } & 347 \text { LFLLHNILNNPEIKVRVYEELDRVLPSPEDTITPQLLLELKYLRACIVESLRMTPVAPNV } \\ \text { D. pulex } & 343 \text { LFLLHNIVSNPEVKARVYEELDRVLYSSHVTITPQLLLELKYLKACVTESLR TPVAPNV } \\ \text { D. melanogaster } 358 \text { LFVLSSVTGDPGAMPRILSEFCE---YRDTNILQDALTNATYTKACIOESYR RPTAFCL } \\ \text { B. mori }\end{array}$

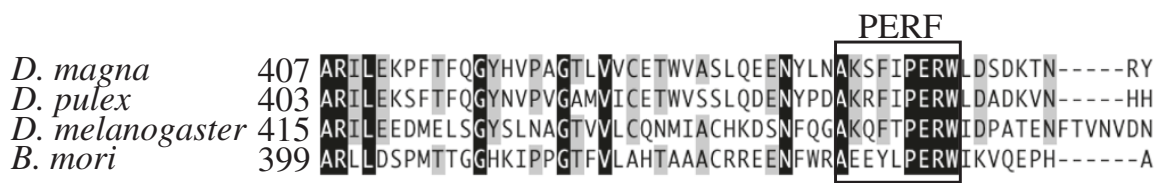

Heme-binding

D. magna 462 PFLAVPFGVGRRMCPGKRIAENEMLIITAKLLRAFDISFHKPLEQVYKFLISPKGPINVI

D. pulex 458 PFLAVPFGVGRRMCPGKRIAEHEMLIITAKLLQTFDMSFQKPLEQVYKFLISPKGPIRAT

D. melanogaster 475 ASIVVPFGVGRRSCPGKRFVEMEVVLLLAKMVLAFDVSFVKPLETEFEFLLAPKTPLSLR

B. mori 453 YSLVAPFGRGRRMCPGKRFVELELHLLLAKIMQKWRVEFDGELDIQFDFLLSAKSPVTLR

Figure 1 Alignments of predicted amino acid sequences of (A) neverland and (B) shade of Daphnia magna with other arthropod species, Daphnia pulex, Drosophila melanogaster and Bombyx mori. D. magna 1 and D. magna 2 indicate the two neverland paralogues, nvd 1 and nvd 2, respectively. The conserved Rieske [2Fe-2S] domain and the non-heme Fe (II) motif in nvd and conserved domain and motifs of the P450 family in shd are shown in boxes. Asterisks show the evolutionally conserved amino acids in nvd. Entries in a black background indicate identities.

In the case of shd, no paralogous genes were identified in the D. magna database. In agreement with the arthropod phylogenetic trend, tick shd is the most ancestral followed by daphnids shd, and insects' shd being the most descendant (Figure 2B). As seen in nvd and shd, the obtained D. magna Cyp18a1 pairs with $D$. pulex Cyp18a1 (Figure 2C) showing a close relationship. 


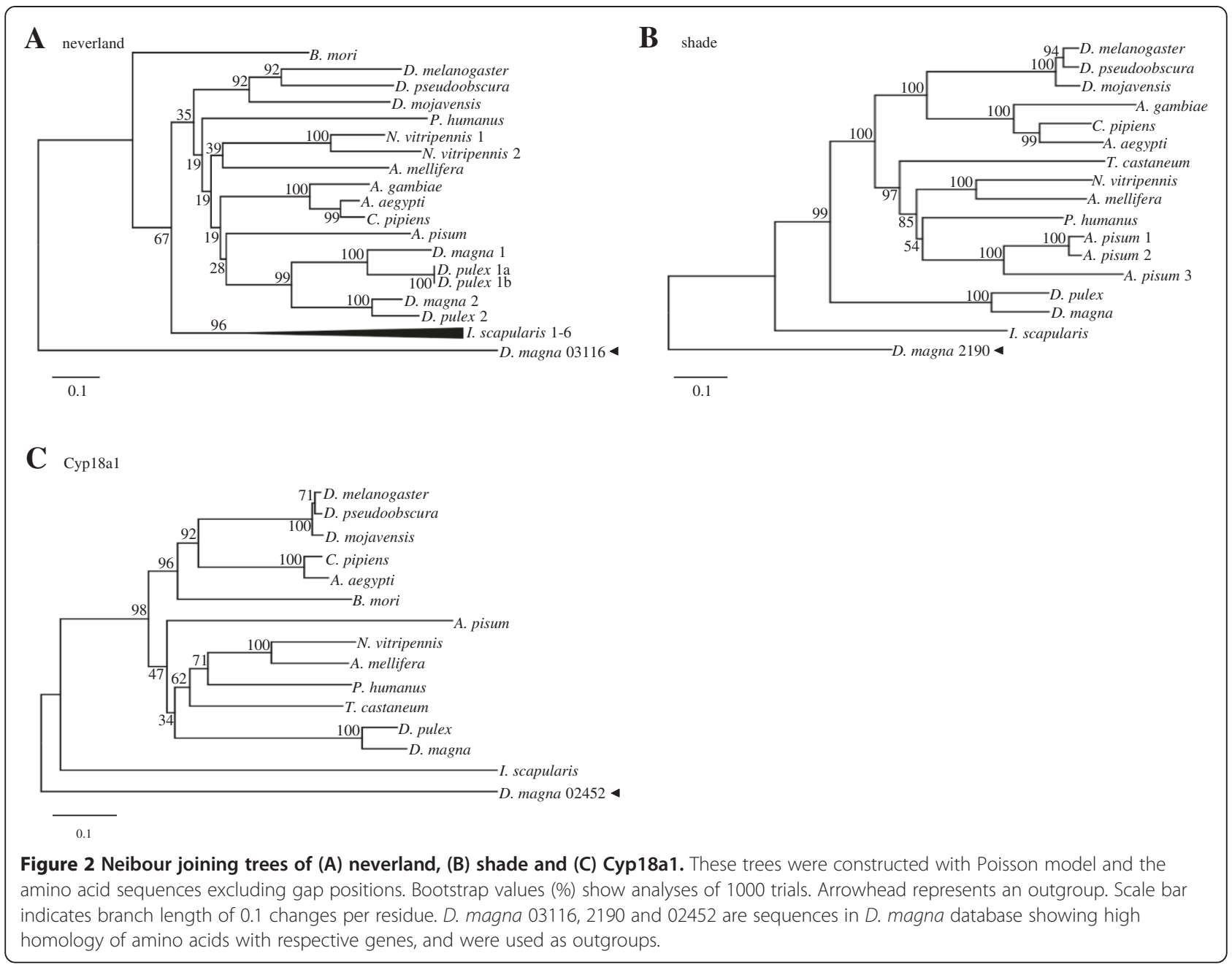

Time-course and tissue-specific expression analyses of neverland, shade, Cyp18a1, ECR and USP during intermoulting period

Adult D. magna has a moulting cycle of three days under current conditions, which takes $80 \mathrm{~h}$ on average between two consecutive moultings. Investigation of the expression profiles of the genes in the ecdysteroids synthesis or inactivation pathway during the intermoulting period was performed by quantitative real-time PCR. $n v d 1, n v d 2$ and shd were highly expressed in the early intermoulting period at 0-10 hours after moulting (ham) (Figure 3A-C). The lowest expression level of $n v d 1$ was reached at 20 ham, and it increased at 70 ham. In contrast, the expression level of $n v d 2$ did not change significantly after the abrupt drop at 20 ham. The expression level of shd gradually decreased until $20 \mathrm{ham}$. Thereafter, it stayed at a rather constant level. Cyp18a1 was also highly expressed at 0 ham, and its expression declined to the lowest level by 30 ham (Figure 3D). At the end of the moulting cycle, Cyp18a1 expression showed a steep rise at 80 ham. The expression level of $E c R$ was relatively high until 10 ham and after 50 ham (Figure 3E). The expression level of USP was highest at 0 ham but decreased to the lowest level by 10 ham after which it no longer shifted (Figure 3F).

A synthetic and target organs/tissues for ecdysteroids are unknown in daphnids. Therefore, tissue-specific expression of genes in the ecdysteroids synthesis or inactivation pathway was analysed in the major organs such as the ovary, gut and remaining tissues (i.e., tissues other than the ovary and gut) by quantitative real-time PCR. $n v d 1$ was strongly expressed in the gut (Figure 4A). $n v d 2$ expression was also observed primarily in the gut, but there was no significant difference in expression levels among tissues (Figure 4B). shd was predominantly expressed in the gut, and its expression was also detected in the ovary and remaining tissues (Figure 4C). Alternatively, the expression level of Cyp18a1 was relatively low in the gut (Figure 4D). The expression level of Cyp18a1 was the highest in the remaining tissues, but not significantly higher than in the ovary or gut. $E c R$ and USP expression were detected in all tissues examined, 

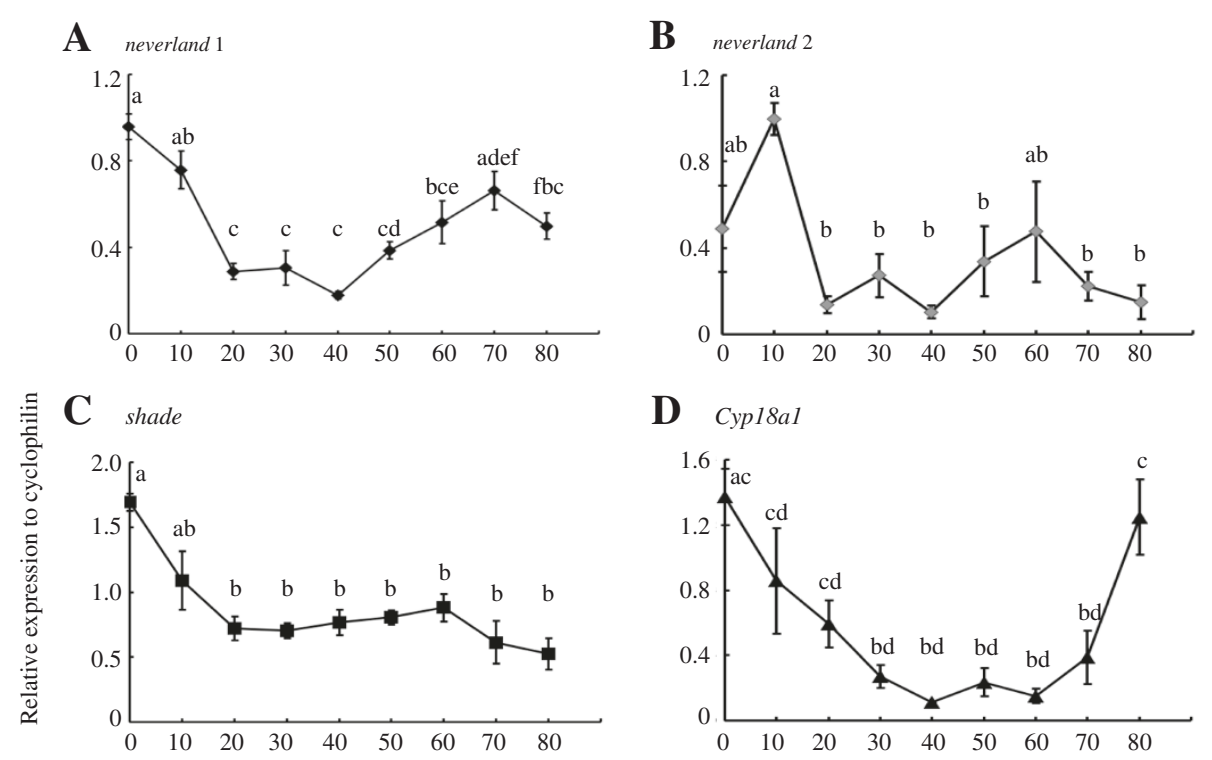

D Cyp18a1
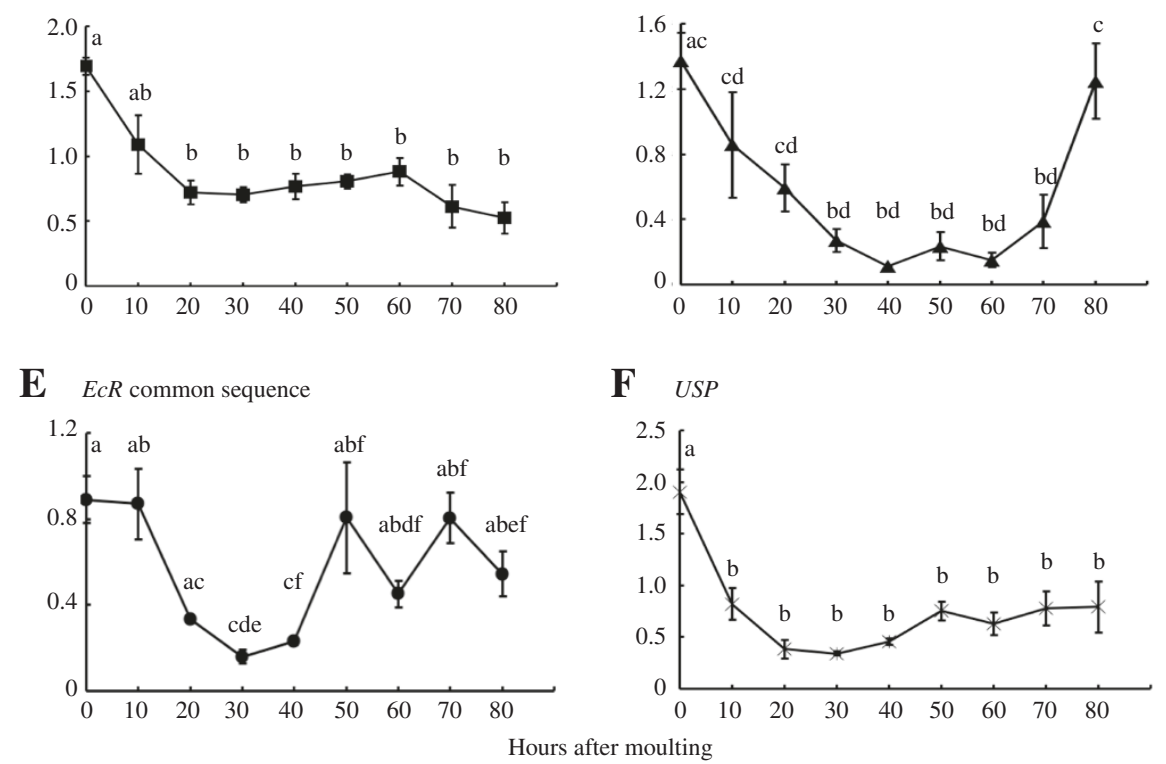

Figure 3 Expression profiles relative to cyclophilin. (A) neverland 1, (B) neverland 2, (C) shade, (D) Cyp18a1, (E) EcR common sequence and (F) USP in the intermoulting period of adult female D. magna. 0 hour after moulting (ham) refers to the time immediately after moulting and 80 ham represents the time before the next moulting. Bars represent standard errors. Different letters indicate significant differences among time points. $P<0.05$ (ANOVA followed by the Tukey-Kramer test).

and the expression levels were highest in the remaining tissues (Figure 4E,F).

\section{Exogenous 20E treatment on adult D. magna in the mid-reproductive cycle}

To understand the importance of fluctuation of the ecdysteroids titre on moulting and ovulation, adult female D. magna were treated with exogenous $20 \mathrm{E}$ in the middle of the reproductive cycle, which is a day before moulting and ovulation occur. After this treatment, unusual phenotypes were observed at a significantly higher rate $(P<0.001)$ : normal moulting without ovulation or neither moulting nor ovulation (Figure 5$)$. In the $20 \mathrm{E}$-treated group, only $34 \%$ of 128 individuals underwent normal moulting followed by ovulation whereas 99\% of 85 control individuals moulted and ovulated normally. The most frequent phenotype appeared in $53 \%$ of the 20E-treated individuals. Those individuals neither moulted nor ovulated. In addition, 13\% of the 20E-treated individuals moulted normally but failed to ovulate. The toxic influence seemed minimal as none of the individuals died within 48 hours after the treatment.

The ovary and carapace were then analysed histologically. The whole ovary was full of yolk granules in control individuals (Figure 6A,B). The meiotic phase of the eggs was also examined. In the ovarian eggs of $D$. pulex, the nuclear envelope degenerates and becomes invisible at the point of moulting [25]. This was confirmed in D. magna. The nuclear envelope was invisible in control individuals before moulting (Figure 6C). After moulting, a meiotic apparatus was prominently visible in the ovarian egg (Figure 6D) and ovulated egg (Figure 6E). In comparison, yolk granules were also present in the ovary of 20E-treated individuals (Figure 6F,G). However, the nuclear envelope did not degenerate in the 20E-treated individuals that failed to moult (Figure 6H). Interestingly, the meiotic apparatus was observed in the ovarian egg of those 20E-treated individuals that failed to ovulate after normal moulting (Figure 6I). As a result, treatment with 20 E caused D. magna to be unable to moult and/or 
A $n v d 1$

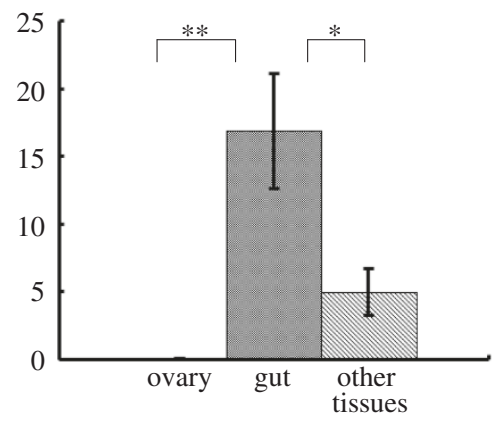

C shade

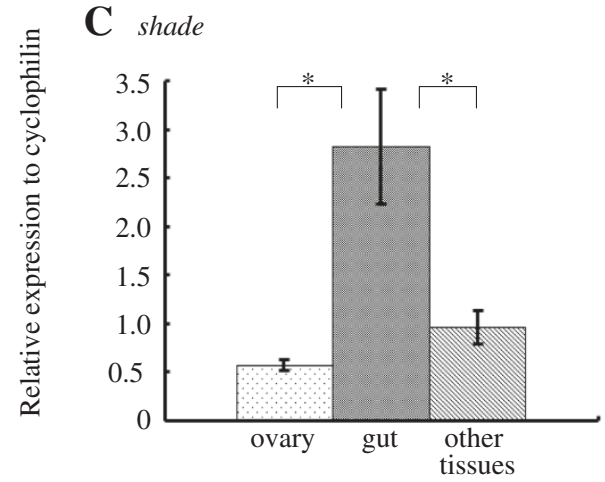

E EcR common sequence

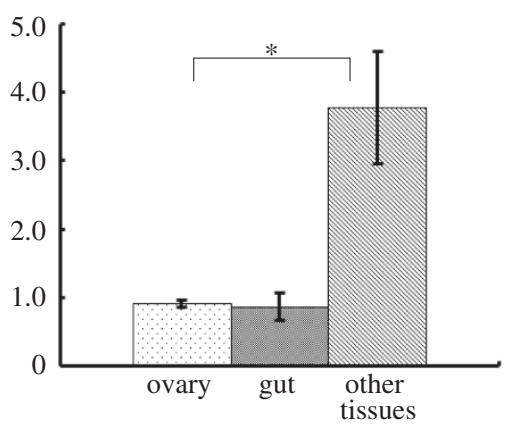

B $n v d 2$

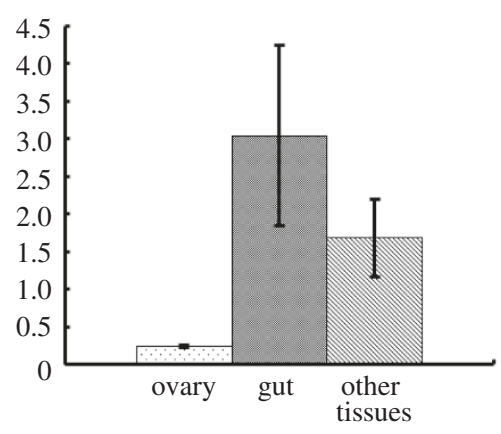

D Cyp18al

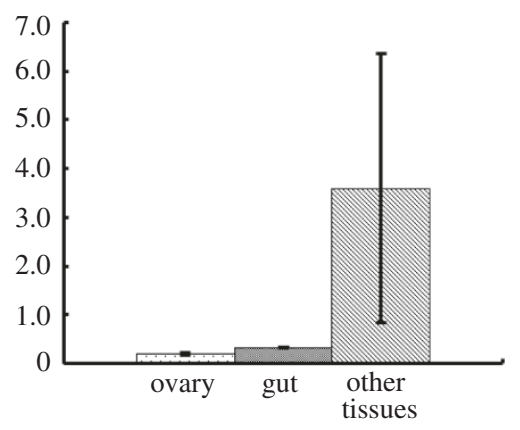

F USP

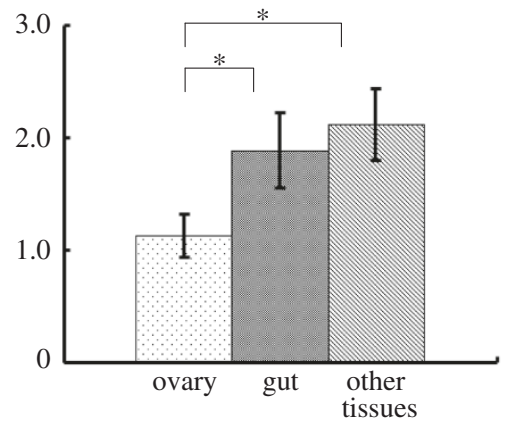

Figure 4 Relative expression to cyclophilin. (A) nvd 1, (B) nvd 2, (C) shd, (D) Cyp18a1, (E) EcR common sequence and (F) USP in D. magna adult female tissues. The ovary, gut and remaining tissues were excised at 70 hours after moulting in the intermoulting period. Bars represent standard errors. ${ }^{*} P<0.05{ }^{* *} P<0.01$ (ANOVA followed by the Tukey-Kramer test).

ovulate, and it ceased meiotic division at the time when the nuclear envelope breaks or meiotic metaphase. A new carapace, which was present in the control before moulting (Figure 6J,K), was also formed in the 20Etreated individuals (Figure 6L,M).

\section{Discussion}

Genes in ecdysteroids synthesis or inactivation pathway are evolutionary conserved in $D$. magna

The moulting hormones, ecdysone and 20E, have long been known to play critical roles in moulting and development of insects and crustaceans [26]. The final product of the ecdysteroids synthesis pathway, 20E, actively interacts with EcR/USP heterodimeric receptor in D. magna [16]. This indicates that the binding of $20 \mathrm{E}$ to EcR in arthropod species can activate the ecdysteroids signalling pathway. Although there is physiological evidence indicating that crustaceans synthesise 20E from cholesterol, the molecular basis of 20E synthesis in crustaceans is not fully understood. Recently, orthologues of the insect Halloween genes encoding P450 enzymes for ecdysteroids biosynthesis such as spook, disembodied, phantom, shadow and shade were obtained by searching the $D$. pulex database [17]. We newly identified genes covering the ecdysteroids synthesis or inactivation pathway, i.e. not only these P450 genes but also nvd and Cyp18a1 in the D. magna EST 


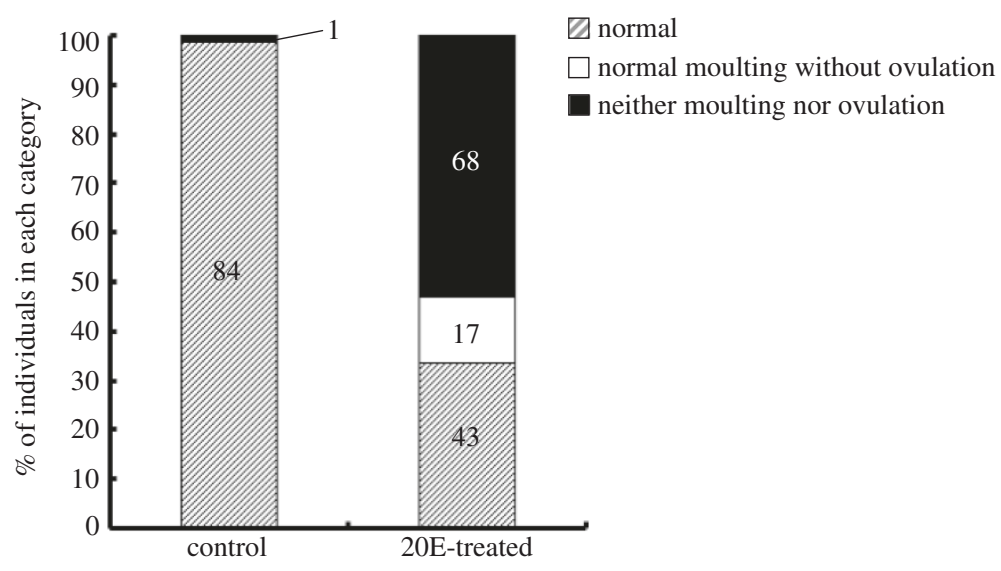

Figure 5 Percentage of adult female $D$. magna categorised into different phenotypic classes after treatment with exogenous 20-hydroxyecdysone (20E) $(N=128)$ or ethanol as the control $(N=\mathbf{8 5})$. Values in bars represent the number of $D$. magna used.

database. The present study focused on nvd, which catalyses the first step in the biosynthesis of ecdysteroids [12], shade mediating the hydroxylation of ecdysone to $20 \mathrm{E}$ [13], and Cyp18a1, a key enzyme for 20E inactivation [14] in insects. Our cloning and phylogenetic analyses showed that nvd and shd as well as Cyp18a1 genes are evolutionary conserved in $D$. magna. This suggests that $D$. magna possesses ecdysteroids synthesis and inactivation pathways similar to those conserved in insects. This result is in agreement with recent phylogenetic analysis suggesting that these genes appeared in arthropods before the radiation of insects. Interestingly, we identified two different paralogues of the $n v d$ gene in $D$. magna. Our phylogenetic analysis indicates that gene duplication gave rise to these two different nvd paralogues, nvd1 and nvd2 in daphnids. This duplication is thought to have occurred after the divergence of daphnids and insects but before the split of $D$. magna and D. pulex. We also identified the two nvd1 paralogues, nvd1a and nvd1b, in D. pulex. In the $D$. pulex linage, a number of gene duplications occurred [27]. These duplication events are thought to have resulted in a complex lifecycle of daphnids.

\section{Cyp18a1 might regulate the decline of ecdysteroids titre before moulting}

In crustaceans including D. magna, shrimp and crab species, ecdysteroids titre shows a single peak during the intermoulting period $[4,28,29]$. In contrast, ecdysteroids titre in insect larvae fluctuates in more complex patterns during embryogenesis and larval development. These rises and falls of ecdysteroids at specific times are mandatory for normal moulting and metamorphosis in insects [30]. Cyp18a1 inactivates 20E through 26-hydroxylation in insects to lower the ecdysteroid titre [14]. Gene expression analysis during the intermoulting period in D. magna showed that the relative expression of Cyp18a1 changed conversely with the fluctuation in ecdysteroids titre (Figure 3D). In other words, the ecdysteroids titre is high in the mid-moulting cycle when Cyp18a1 expression is low, but is low at the end of the moulting cycle when Cyp18a1 expression is high. This result suggests that Cyp18a1 possibly regulates the decline of the ecdysteroids titre before moulting in D. magna.

Relative expression analysis of shd and Cyp18a1 showed different patterns (Figure 3C,D). Each P450 gene in the ecdysteroids synthesis pathway exhibits a different expression pattern in insect larvae, and it varies among species [24,31-34]. Therefore, having a unique expression pattern for each of the genes appears to be a common phenomenon. Such variable expression patterns reflect which gene is responsible for a rate-limiting step or is a target of prothoracicotropic hormone (PTTH), which induces ecdysone synthesis in insects [33].

\section{Ecdysone might be synthesised in the gut, judged by the expression of neverland}

In insect larvae, expression of $n v d$ is exclusively detected in the ecdysone synthetic organ [12]. On the other hand, shd has been observed in a range of 20E-target tissues/ organs $[13,24,31]$. In adult D. melanogaster, $n v d$ is expressed in the ovary where ecdysone is produced [12]. A gene encoding phantom, which participates in ecdysone synthesis, in kuruma prawn is expressed in the Y-organ [18]. D. magna $n v d 1$ was primarily expressed in the gut of the adult, suggesting that ecdysone is possibly synthesised in the gut. The expression of shd was detected in the ovary as well as in the gut at 70 ham when oogenesis had been promoted in the ovary for upcoming ovulation, and the eggs had formed inside. Such spatial expression of shd indicates that ecdysone targets the ovary besides the gut and remaining tissues. Ecdysteroids might function in ovarian egg maturation in D. magna. 


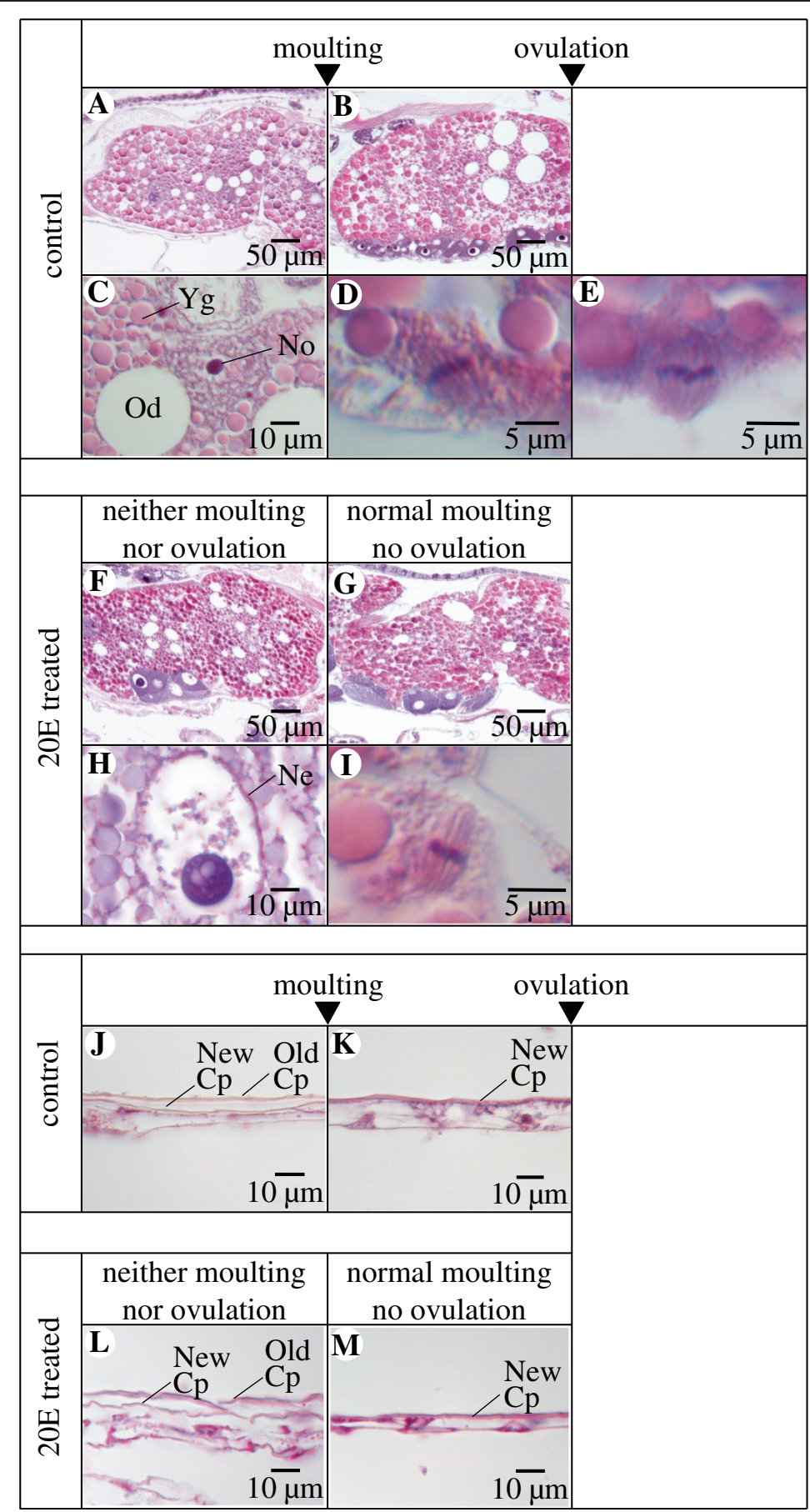

Figure 6 Histological analysis of the ovary and carapace. (A,B) Ovarian eggs in a control individual. (C) The nuclear envelope was invisible in controls before moulting. (D,E) A meiotic apparatus found in ovarian and ovulated eggs of controls, respectively, and (F,G) ovarian eggs of a 20E-treated individual. (H) The nuclear envelope had not degenerated in the ovarian egg in the 20E-treated individual that neither moulted nor ovulated. (I) A division apparatus appeared and chromosomes aligned at the metaphase plate in the 20E-treated individual that moulted normally but did not ovulate. This meiotic apparatus at metaphase was also seen in the control eggs released into the brood pouch immediately after ovulation. However, in the 20E-treated individuals, meiosis stopped at metaphase and did not proceed. (J,K) Carapace formation in controls and (L,M) 20E-treated individuals. (J) A carapace layer with a newly formed carapace underneath the old one formed before moulting in the control. (K) A new carapace was observed immediately after moulting in the control. (L) A new carapace was also formed in the 20E-treated individual that neither moulted nor ovulated, and (M) the 20E-treated individual that moulted normally but did not ovulate. Yg: yolk granule, No: nucleolus, Od: oil droplet, Ne: nuclear envelope, Cp: carapace. Tissue sections of exogenous 20E-treated and the control adult female D. magna were stained with hematoxylin and eosin. 


\section{The decline of ecdysteroids level before moulting is required for ensuing moulting and ovulation}

Ecdysteroids stimulate vitellogenesis in most decapod species [35]. On the other hand, 20E inhibits vitellogenesis in D. magna [36]. In support of this, endogenous ecdysteroids titre is low when moulting and ovulation are about to occur in D. magna [11]. Ovulation occurs immediately after moulting when ecdysteroids titre has decreased. As such a correlation between ecdysteroids titre, moulting and reproductive events exists, we hypothesised that the fluctuation of ecdysteroids titre plays a key role in the endocrine systems regulating the reproductive and moulting cycles. Therefore, we analysed the effect of 20E-treatment on moulting and ovulation in D. magna. Treatment with $20 \mathrm{E}$ from the middle of the moulting cycle inhibited moulting and/or ovulation. This indicates that a decrease of ecdysteroids at the end of the reproductive cycle is required for moulting and ovulation. Indeed, Cyp18a1 was highly expressed at the end of the moulting cycle. Histological analyses revealed that yolk granules existed in the ovary of both the control and 20E-treated D. magna. This implies that suppressed ovulation might not have been caused by inhibition of vitellogenesis by a high $20 \mathrm{E}$ titre. In the present study, those individuals that did not moult after treatment with $20 \mathrm{E}$ produced a new carapace underneath the old one. Therefore, 20E treatment did not affect the process of cuticle deposition.

Recently, a meiosis-like process of parthenogenetic oogenesis was documented in D. pulex [25]. In the present study, the nuclei-germinal vesicles broke down (GVBD: germinal vesicle breakdown), chromosomes condensed, and the nuclei were invisible before moulting in the ovarian eggs of control D. magna, as observed in D. pulex. In the ovary of 20E-treated individuals showing neither moulting nor ovulation, the meiotic process had stopped before GVBD. Although the ovary contained as many yolk granules as the control ovary, 20E inhibited the entry of the ovarian eggs into GVBD, which marks the onset of meiosis.

A meiotic apparatus at metaphase was observed soon after moulting and soon after ovulation in the control eggs in D. magna (Figure 6D,E). Such a meiotic apparatus at metaphase was also observed in the eggs of 20E-treated individuals that moulted normally but failed to ovulate (Figure 6I). In the 20E-treated individuals, however, meiosis stopped at metaphase and did not proceed further even after enough time had passed for meiosis to progress (Figure 6I). Such a terminated meiotic process might correspond to a failure in meiotic reinitiation. The first reinitiation appears as GVBD and the second as anaphase. Furthermore, meiotic reinitiation is correlated with the ecdysteroids titre in prawn (Palaemon serratus) and locust (Locusta migratoria) [37]. The present results suggest that the 20E titre might also be responsible for meiotic reinitiation in D. magna. Ovulation never occurs unless moulting precedes it. The time interval between moulting and ovulation is precisely 13 minutes in $D$. pulex at $18^{\circ} \mathrm{C}$ [25]. Therefore, moulting is expected to act as an ovulation trigger. There must be coordinated regulation between moulting and ovulation. Our results are compatible with this idea, but moulting alone is not enough to initiate ovulation. Some regulatory mechanisms might be present to promote ovulation after moulting. Alternatively, some inhibitory mechanisms might also be present for ovulation of abnormal eggs.

In Drosophila, neural signalling with octopamine, which causes oviduct-muscle contraction, controls ovulation $[38,39]$. In decapod crustaceans, octopamine in the lobster Homarus americanus also stimulates muscle contraction of ovarian walls [3]. Neural signalling besides a direct endocrine pathway could possibly drive the regulation of ovulation in D. magna. That is because ovarian eggs likely require some mechanical force, such as muscle contraction, to be released into the brood pouch. This suggests that 20E might affect ovulation through muscle contraction.

\section{Conclusions}

In conclusion, we identified genes in the ecdysteroids synthesis or inactivation pathway in D. magna. Decline of the ecdysteroids level at the end of the reproductive cycle is necessary for ensuing moulting and ovulation. Our gene expression analyses suggest that fluctuation of the ecdysteroids titre during the reproductive cycle might be regulated by the expression of Cyp18a1. Judging by the predominant expression of $n v d 1$ in the gut, we also propose that ecdysone might be synthesised in the $D$. magna gut although further studies are required to identify the specific site of ecdysteroidogenesis in the gut. Gene identification and expression analyses in ecdysteroids synthesis or inactivation pathways provide a concrete basis for understanding the contribution of ecdysteroids signalling in the highly coordinated reproductive system of crustaceans.

\section{Materials and methods \\ Animals}

Daphnia magna (NIES strain) obtained from the National Institute for Environmental Studies (NIES; Tsukuba, Japan) were maintained in M4 medium [40] at $20 \pm 1^{\circ} \mathrm{C}$ under a 14-hour:10-hour light:dark photoperiod. Chlorella vulgaris was added daily into each culture. For experiments, each animal was incubated under the same conditions mentioned above but in $50 \mathrm{ml}$ of M4 medium individually. The culture medium was substituted every three days. Under these conditions, D. magna produces female offspring every three days. 
Table 1 Primer sequences used for gene cloning

\begin{tabular}{lll}
\hline Genes & Forward & Reverse \\
\hline neverland 1 & ATGGATGTCTTTTCGTTTCAAATCC & GGTGTAACCGTCTCGAAG \\
neverland 2 & GGACGCGTATTGCCCTCATTTGGA & TAATCCCGGCCCGATTTGTTGGCT \\
3' RACE & ATGATCGTGTGGAGTTGGGA & TाTGGCGTTCTGGATCTCCAG \\
neverland 1 & & \\
Cyp18a1 & GCCTCCATCATGAACTACGCCTATTCGG & \\
& GCGACGGCCGTGAGAAAATGGAG & \\
\hline
\end{tabular}

\section{Cloning and phylogenetic analysis}

Total RNA extracted from adult female D. magna using the RNAqueous Kit (Life Technologies, Carlsbad, CA, USA) was reverse transcribed with Super Script III (Life Technologies). To obtain gene fragments of $n v d$ and Cyp18a1, oligonucleotide primers were designed based on the sequences of the D. magna EST database [41] (Table 1). PCR was performed with Platinum Taq DNA Polymerase High Fidelity (Life Technologies). The amplified products were cloned into pGEM-T easy (Promega, Tokyo, Japan), and sequenced using the BigDye Terminator Cycle Sequencing Kit (Life Technologies). Based on the sequence information of the obtained PCR products, the 5' and 3' ends of $n v d 1$ and Cyp18a1 sequences were amplified using a rapid amplification cDNA ends (RACE) kit (Gene Racer Kit, Life Technologies) with Platinum Taq High Fidelity (Life Technologies). The obtained two nvd and Cyp18a1 sequences were assigned the following accession numbers [DDBJ: $n v d 1$ : AB839171, nvd2: AB839172 and Cyp18a1: AB839173]. Sequence of shd of $D$. magna was available in the NCBI database (AB257771.1). Sequences of EcR and USP were defined in our previous study (AB274819-24) [16].

The amino acid sequences of $n v d$, shd and Cyp18a1 in other arthropod species were identified in euGenes/ Arthropod genomes (Indiana University, http://arthropods. eugenes.org/arthropods/). The amino acid sequences were aligned with MEGA5 software using MUSCLE method with the default options [42]. Neighbour joining trees were constructed from these alignments using Poisson model with bootstrap analyses of 1000 replicates along with complete deletion options.

\section{Quantitative PCR}

Whole body adult $D$. magna samples were prepared at nine time points with 10-hour intervals during an 80-hour intermoulting period. For the tissue-specific gene expression analysis, adult D. magna at 70 ham was dissected, and ovary, gut and the remaining tissues were separately obtained. Three replicates were prepared for each time point for each experiment. Total RNA was extracted using the RNAqueous-Micro Kit (Life Technologies). cDNA was synthesised from $0.5 \mu \mathrm{g}$ of total RNA using Super Script III (Life Technologies) with random primers. PCR was conducted with SYBR-Green PCR Master Mix (Life Technologies) using the 7000 real-time PCR system (Life Technologies). The primer sequences used for the quantitative PCR analyses are shown in Table 2. To detect $E c R$, a common sequence region of three $E c R$ genes was utilized to design the primers. Among four housekeeping genes (cyclophilin, Ltd4ds, LBT and L32), D. magna cyclophilin was selected as a reference gene calculated with geNorm [43] and NormFinder [44]. Statistical analyses of gene expression at each time point were performed by ANOVA followed by the Tukey-Kramer post-hoc test using Excel 2004 (Microsoft Corp., Redmond, WA) with an add-in software Statcel 2 [45].

Table 2 Primer sequences used for quantitative real-time PCR

\begin{tabular}{lll}
\hline Genes & Forward & Reverse \\
\hline cyclophilin & GACTTCCACCAGTGCCATT & AACTTCCATCGCATCATCC \\
neverland 1 & AGCACAAGGCGGGAAGAGT & GCTTCCCATTTCACCTTCCA \\
neverland 2 & CGTCGGTGACTGCATCGA & TGCCGTCGTTCCCATTG \\
shade & GACTGCTGAAGGCGTTGACA & CGGCTGCCACTAGGTCGATA \\
Cyplea1 & TACCCGATCGTCGGTTACCT & GAGCGCCGTCAGCTCTTC \\
ECR common region & GAGGCGCTGCAGGCTTAC & GAGTTGGCAAACTCCGTCATC \\
USP & GTTGGAGTCAAGGATGGTATCGT & AGCCGAGTTCCGGGGAT \\
\hline
\end{tabular}




\section{Treatment with exogenous ecdysteroid}

A stock solution of $10 \mathrm{mM} \mathrm{20E} \mathrm{(Sigma,} \mathrm{St.} \mathrm{Luis,} \mathrm{MO,}$ USA) dissolved in ethanol was kept at $-20^{\circ} \mathrm{C}$ until use. This stock solution was directly added to each $50 \mathrm{ml}$ of M4 medium containing one adult female $D$. magna at two days after moulting $(N=128)$. The final concentration was $0.5 \mu \mathrm{M} 20 \mathrm{E}$. Ethanol $(0.0001 \%)$ served as the control $(N=85)$. The number of individuals was counted for three phenotypes in the following order of severity: normal moulting and ovulation (no effect), normal moulting following no ovulation (moderate effect), or neither moulting nor ovulation (severe effect). Differences in the incidence between the control and 20E-treated animals were analysed with the Mann-Whitney $U$ test using Excel 2004 (Microsoft Corp.) with Statcel 2 [45]. For tissue sectioning, the 20E-treated and control animals were fixed in Bouin's fluid overnight. The samples dehydrated in ethanol and xylene were embedded in paraffin. Serial sections of $5 \mu \mathrm{m}$ thickness were subjected to haematoxylin and eosin staining. The sections were studied under a microscope (Axioplan2, ZEISS, Oberkochen, Germany) and the images were processed with callSens Standard, version 1.3 (Olympus, Tokyo, Japan). Six individuals of the 20Etreated animals were observed for each of the three phenotypes. Three control animals were examined at three stages: before moulting (sampled after the release of a brood), soon after moulting and before ovulation (sampled within 15 minutes after moulting) and soon after ovulation (sampled when ovulation finished).

\section{Abbreviations \\ Cyp450: CyctochromeP450; EcR: Ecdysone receptor; GVBD: Germinal vesicle breakdown; Ham: Hours after moulting; Nvd: Neverland; shd: Shade; USP: Ultra spiracle; 20E: 20-hydroxyecdysone. \\ Competing interests \\ The authors declared that they have no competing interests. \\ Authors' contributions \\ ES performed experiments, analysed data, and wrote the manuscript. YO and $\mathrm{TI}$ conceived the study. HM participated in the experimental design and phylogenetic analysis. $\mathrm{CH}, \mathrm{KT}$, SM participated in the interpretation of data. $\mathrm{YO}, \mathrm{HM}, \mathrm{CH}$ and $\mathrm{TI}$ also helped with the manuscript. All authors read and approved the final manuscript.}

\section{Acknowledgements}

D. magna and D. pulex sequence data were provided by The Center for Genomics and Bioinformatics at Indiana University which is supported in part by the METACyt Initiative of Indiana University, funded in part through a major grant from Lilly Endowment, Inc., and distributed via wFleaBase in collaboration with Dr. Donald Gilbert and the Daphnia Genomics Consortium https://wiki.cgb.indiana.edu/display/DGC/Home. This work was partly supported by a Japan Society for the Promotion of Science (JSPS) Grant-in-Aid to ES (No. 26.04101), and by grants from the Ministry of Education, Culture, Sports, Science and Technology (TI), the Ministry of the Environment of Japan (TI), a grant from the National Institute for Basic Biology (TI), and the Research Council of Norway (project 221455) -.Adverse Outcome Pathways for Endocrine Disruption in Daphnia magna, a conceptual approach for mechanistically-based risk assessment (TI).

Received: 9 June 2014 Accepted: 11 August 2014 Published: 19 August 2014

\section{References}

1. Kenny NJ, Quah S, Holland PW, Tobe SS, Hui JH: How are comparative genomics and the study of microRNAs changing our views on arthropod endocrinology and adaptations to the environment? Gen Comp Endocrinol 2013, 188:16-22.

2. Bliss DE: Ecological aspects of reproduction. In The Biology of Crustacea. Edited by Vernberg FJ, Vernberg WB. New York: Academic Press, Inc; 1983:179-191.

3. Swetha CH, Sainath SB, Reddy PR, Reddy PS: Reproductive endocrinology of female crustaceans: perspective and prospective. J Marine Sci Res Dev 2011, S3:2.

4. Gunamalai $V$, Kirubagaran R, Subramoniam T: Hormonal coordination of molting and female reproduction by ecdysteroids in the mole crab Emerita asiatica (Milne Edwards). Gen Comp Endocrinol 2004, 138:128-138.

5. Shyama SK: Studies on moulting and reproduction in the prawn Macrobrachium idella (hellar). Mahasagar-Bull Natl Inst Oceanog 1987, 20:15-21.

6. Nijhout HF: Insect Hormones. Princeton University Press: Princeton; 1994.

7. Ables ET, Drummond-Barbosa D: The steroid hormone ecdysone functions with intrinsic chromatin remodeling factors to control female germline stem cells in Drosophila. Cell Stem Cell 2010, 7:581-592.

8. König A, Yatsenko AS, Weiss M, Shcherbata HR: Ecdysteroids affect Drosophila ovarian stem cell niche formation and early germline differentiation. EMBO J 2011, 30:1549-1562.

9. Agawa Y, Sarhan M, Kageyama Y, Akagi K, Takai M, Hashiyama K, Wada T, Handa H, Iwamatsu A, Hirose S, Ueda H: Drosophila Blimp-1 is a transient transcriptional repressor that controls timing of the ecdysone-induced developmental pathway. Mol Cell Biol 2007, 27:8739-8747.

10. Akagi $\mathrm{K}$, Ueda H: Regulatory mechanisms of ecdysone-inducible Blimp-1 encoding a transcriptional repressor that is important for the prepupal development in Drosophila. Dev Growth Deffir 2011, 53:697-703.

11. Martin-Creuzburg D, Westerlund SA, Hoffmann KH: Ecdysteroid levels in Daphnia magna during a molt cycle: Determination by radioimmunoassay (RIA) and liquid chromatography-mass spectrometry (LC-MS). Gen Comp Endocrinol 2007, 151:66-71.

12. Yoshiyama T, Namiki T, Mita K, Kataoka H, Niwa R: Neverland is an evolutionally conserved Rieske-domain protein that is essential for ecdysone synthesis and insect growth. Development 2006, 133:2565-2575.

13. Petryk A, Warren JT, Marques G, Jarco MP, Gilbert LI, Kahler J, Parvy J, Li Y, Dauphin-Villemant C, O'Connor MB: Shade is the Drosophila P450 enzyme that mediates the hydroxylation of ecdysone to the steroid insect molting hormone 20-hydroxyecdysone. Proc Natl Acad Sci U S A 2003, 100:3773-13778.

14. Guittarda E, Blais C, Maria A, Parvy J, Pasricha S, Lumb C, Lafont R, Daborn PJ, Dauphin-Villemant C: CYP18A1, a key enzyme of Drosophila steroid hormone inactivation, is essential for metamorphosis. Dev Biol 2011, 349:35-45.

15. Hopkins PM: Crustacean ecdysteroids and their receptors. In Ecdysone: structures and functions. Edited by Smagghe G. Netherlands: Springer; 2009:73-97.

16. Kato Y, Kobayashi K, Oda S, Tatarazako N, Watanabe H, Iguchi T: Cloning and characterization of the ecdysone receptor and ultraspiracle protein from the water flea Daphnia magna. J Endocrinol 2007, 193:83-194.

17. Rewitz KF, Gilbert LI: Daphnia Halloween genes that encode cytochrome P450s mediating the synthesis of the arthropod molting hormone: Evolutionary implications. BMC Evol Biol 2008, 8:60.

18. Asazuma $H$, Nagata $\mathrm{S}$, Nagasawa $\mathrm{H}$ : Inhibitory effect of molt-inhibiting hormone on phantom expression in the Y-organ of the kuruma prawn, Marsupenaeus japonicus. Arch Insect Biochem Physiol 2009, 70:220-233.

19. Tom M, Manfrin C, Giulianini PG, Pallavicini A: Crustacean oxi-reductases protein sequences derived from a functional genomic project potentially involved in ecdysteroid hormones metabolism-A starting point for function examination. Gen Comp Endocrinol 2013, 194:71-80.

20. Marchal E, Badisco L, Verlinden H, Vandersmissen T, Soest SV, Wielendaele PV, Broeck JV: Role of the Halloween genes, Spook and Phantom in ecdysteroidogenesis in the desert locust, Schistocerca gregaria. J Insect Physiol 2011, 57:1240-1248.

21. Lachaise F, Le Roux A, Hubert M, Lafont R: The molting gland of crustaceans: localization, activity, and endocrine control (a review). J Crustacean Biol 1993, 13:198-234.

22. Lafont $\mathrm{R}$, Mathieu M: Steroids in aquatic invertebrates. Ecotoxicology 2007, 16:109-130. 
23. Zhu XX, Oliver JH, Dotson EM: Epidermis as the source of ecdysone in an argasid tick. Proc Natl Acad Sci U S A 1991, 88:3744-3747.

24. Iga M, Smagghe G: Identification and expression profile of Halloween genes involved in ecdysteroid biosynthesis in Spodoptera littoralis. Peptides 2010, 31:456-467.

25. Hiruta C, Nishida C, Tochinai S: Abortive meiosis in the oogenesis of parthenogenetic Daphnia pulex. Chromosome Res 2010, 18:833-840.

26. Subramonian T: Crustacean ecdysteroids in reproduction and embryogenesis. Comp Biochem Physiol C 2000, 125:135-156.

27. Colbourne JK, Pfrender ME, Gilbert D, Thomas WK, Tucker A, Oakley TH, Tokishita S, Aerts A, Arnold GJ, Basu MK, Bauer DJ, Cáceres CE, Carmel L, Casola C, Choi J, Detter JC, Dong Q, Dusheyko S, Eads BD, Fröhlich T, GeilerSamerotte KA, Gerlach D, Hatcher P, Jogdeo S, Krijgsveld J, Kriventseva EV, Kültz D, Laforsch C, Lindquist E, Lopez J, et al: The ecoresponsive genome of Daphnia pulex. Science 2011, 331:555-561.

28. Soumoff $C$, Skinner DM: Ecdysteroid titers during the molt cycle of the blue crab resemble those of other Crustacea. Biol Bull 1983, 165:321-329.

29. Huberman: A shrimp endocrinology. A review. Aquaculture 2000, 191:191-208.

30. Gilbert LI: Insect Endocrinology. 1st edition. London: Academic; 2012.

31. Rewitz KF, Rybczynski R, Warren JT, Gilbert LI: Identification, characterization and developmental expression of Halloween genes encoding P450 enzymes mediating ecdysone biosynthesis in the tobacco hornworm, Manduca sexta. Insect Biochem Mol Biol 2006, 36:188-199.

32. Ono H, Rewitz KF, Shinoda T, Itoyama K, Petryk A, Rybczynski R, Jarcho M, Warren JT, Marqués G, Shimell MJ, Gilbert LI, O'Connor MB: Spook and Spookier code for stage-specific components of the ecdysone biosynthetic pathway in Diptera. Dev Biol 2006, 298:555-570

33. Yamanaka N, Honda N, Osato N, Niwa R, Mizoguchi A, Kataoka H: Differential regulation of ecdysteroidogenic $\mathrm{P} 450$ gene expression in the silk moth, Bombyx mori. Biosci Biotechnol Biochem 2007, 71:2808-2814.

34. Niwa R, Namiki T, Ito K, Shimada-Niwa Y, Kiuchi M, Kawaoka S, Kayukawa T, Banno Y, Fujimoto Y, Shigenobu S, Kobayashi S, Shimada T, Katsuma S, Shinoda T: Non-moulting glossy/shroud encodes a short-chain dehydrogenase/reductase that functions in the 'Black Box' of the ecdysteroid biosynthesis pathway. Development 2010, 137:1991-1999.

35. Nagaraju GPC: Reproductive regulators in decapod crustaceans: an overview. J Exp Biol 2010, 214:3-16.

36. Hannas BR, Wang YH, Thomson S, Kwon G, Li H, LeBlanc JA: Regulation and dysregulation of vitellogenin mRNA accumulation in daphnids (Daphnia magna). Aquat Toxicol 2011, 101:351-357.

37. Lanot R, Clédon P: Ecdysteroids and meiotic reinitiation in Palaemon serratus (Crustacean Decapoda natantia) and in Locusta migratoria (Insecta orthoptera). A comparative study. Invert Reprod Dev 1989, 16:169-175

38. Lee HG, Seong CS, Kim YC, Davis RL, Han KA: Octopamine receptor OAMB is required for ovulation in Drosophila melanogaster. Dev Biol 2003, 264:179-190.

39. Rodríguez-Valentín R, López-González I, Jorquera R, Labarca P, Zurita M, Reynaud E: Oviduct contraction in Drosophila is modulated by a neural network that is both, octopaminergic and glutamatergic. J Cell Physiol 2006, 209:183-198.

40. Elendt BP, Bias WR: Trace nutrient deficiency in Daphnia magna cultured in standard medium for toxicity testing, effects of the optimization of culture conditions on life history parameters of Daphnia magna. Water Res 1990, 24:1157-1167.

41. Kato Y, Kobayashi K, Watanabe H, Iguchi T: Environmental sex determination in the branchiopod crustacean Daphnia magna: deep conservation of a Doublesex gene in the sex-determining pathway. PLoS Genet 2011, 7:e1001345.

42. Tamura K, Peterson D, Peterson N, Stecher G, Nei M, Kumar S: MEGA5: Molecular evolutionary genetics analysis using maximum likelihood, evolutionary distance, and maximum parsimony methods. Mol Biol Evol 2011, 28:2731-2739.

43. Vandesompele J, de Preter K, Pattyn F, Poppe B, van Roy N, de Paepe A, Speleman F: Accurate normalization of real-time quantitative RT-PCR data by geometric averaging of multiple internal control genes. Genome Biol 2002, 3:research0034.
44. Andersen $\mathrm{CL}$, Jensen JL, Ørntoft TF: Normalization of real-time quantitative reverse transcription-PCR data: a model-based variance estimation approach to identify genes suited for normalization, applied to bladder and colon cancer data sets. Cancer Res 2004, 64:5245-5250.

45. Yanai H: Statcel, the Useful Add-in Software Forms on Excel. 2nd edition. Tokyo: OMS; 2004

doi:10.1186/s12983-014-0060-2

Cite this article as: Sumiya et al: Roles of ecdysteroids for progression of reproductive cycle in the fresh water crustacean Daphnia magna.

Frontiers in Zoology 2014 11:60.

\section{Submit your next manuscript to BioMed Central and take full advantage of:}

- Convenient online submission

- Thorough peer review

- No space constraints or color figure charges

- Immediate publication on acceptance

- Inclusion in PubMed, CAS, Scopus and Google Scholar

- Research which is freely available for redistribution 